\title{
The Role of Supraspinatous Ligament Integrity on Adjacent Segment Instability after Lumbar Instrumentation and Laminectomy - A Biomechanical Study in Porcine Model
}

\author{
Po-Liang Lai ${ }^{1}$, Wen-Jer Chen ${ }^{1}$, Lih-Huei Chen', Ching-Lung Tai ${ }^{2}$ \\ 'Department of Orthopaedic Surgery, Chang Gung Memorial Hospital, Taipei, Taiwan \\ 2 Biomechanical Lab, Chang Gung Memorial Hospital, Taipei, Taiwan
}

\section{Introduction}

Instrumented lumbar fusion and laminectomy has been widely accepted for treatment of spondylolisthesis and traumatic spine injury. With improved fusion rates form the use of pedicle screw instrumentation, breakdown of the unfused adjacent motion segment has been identified as a possible cause of postoperative pain and disability $[1,2]$.

Multiple risk factors associated with postoperative adjacent motion segment instability have been reported. The current study focused on the biomechanical effect due to difference between laminectomy level and fusion level.

\section{Materials and Methods}

Eight fresh adult porcine lumber spines (Ll-S1) received a single motion segment instrumentation at L4-L5 using screw-rod system. All the specimens underwent laminectomy in order to simulate the procedure performed on human. All the specimens underwent flexion-extension test under conditions of intact (no laminectomy), partial laminectomy and complete laminectomy in sequence (Fig.1). The intact and partial laminectomy groups preserved the supraspinatous and interspinous ligament at L3-L4 ; the complete laminectomy group removed the entire $\mathrm{L} 4$ spinous process.

The specimens were mounted for biomechanical test using MTS testing machine (Bionix 858). An 8,400 N-mm constant moment was applied to the spine specimen to achieve the flexion and extension motions (Fig.2). The intervertebral displacement at $\mathrm{L} 3-\mathrm{L} 4$ was recorded using a MTS extensometer (Model 632-12F-20). The data of intervertebral displacement was recorded simultaneously during testing by MTS Testor II software. A total of six tests were performed for each porcine specimen. First, the intervertebral displacements of L3-L4 of lumbar spine were measured under conditions of flexion and extension motions respectively. Next, partial laminectomy was performed by removal of the inferior portion of L4 spinous process, and the measurement of the intervertebral displacements of L3-L4 of the lumbar spine in partial laminectomy was repeated again. Finally, the measurements of the lumbar spine in complete laminectomy were carried out using identical testing procedures. The stability of intact, partial laminectomy and complete laminectomy of the lumbar spine were evalúated by comparing the data of intervertebral displacement between L3-L4.

\section{Results}

The mean intervertebral displacements at L3-L4 are shown in Table 1 . The results revealed that there was no statistical difference of intervertebral displacements among three different integrities of posterior elements under extension motion $(\mathrm{P}>0.05)$. However, under flexion motion, the intervertebral displacement on the adjacent disc with complete laminectomy treatment was statistically higher than those with intact or partial laminectomy treatments $(P<0.05)$. Additionally, no difference was found between groups with intact and partial laminectomy treatments.

\section{Discussion}

The typical diagram of intervertebral displacement versus applied moment on superior adjacent segment under flexion motion is shown in Fig 3. The curve demonstrates that the thickness of disc decreased significantly with increasing flexion moment at the initial period. However, the decreasing rate (slope) decreased gradually with increasing flexion moment due to the increase of the intradiscal pressure during testing. The intervertebral displacement of the lumbar with complete laminectomy was significantly higher than those of lumbar with intact or partial laminectomy. This causes the greatest amount of abnormal stress on the superior adjacent motion segment. This result is well corresponding with our hypothesis that the integrity of spinous process-supraspinous ligament-spinous process plays an important role for the neighboring motion segment stability after instrumentation. From the viewpoint of biomechanics, if the decompression level 
overextends than the fusion level, it is more likely to develop adjacent instability. Therefore, if the decompression is localized to lower L4 lamina, the chance of developing adjacent instability is less than decompression including the whole L4 lamina. In the current study, the complete laminectomy means removal of lamina and spinous process of whole L4. Such treatment sacrifices the anchoring point for L3-L4 supraspinatous ligament, which destroys the checking mechanism in bending. This bone-tendon-bone model acts as a tension band for dynamic stability.

\section{Conclusion}

Based on the results, we conclude that sacrificing either the supraspinatous ligament or the anchoring points on spinous processes will cause increased stress at the adjacent unfused segment.

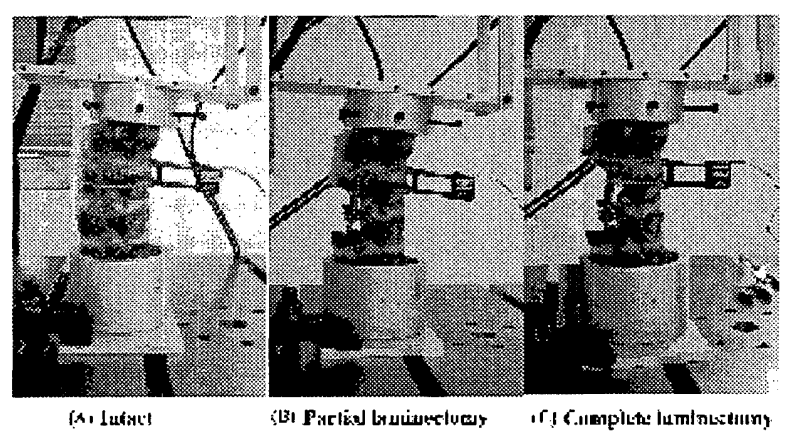

Fig.1 After L4-L5 instrumentation, all porcine lumbar spines underwent (A) intact (no laminectomy), (B) partial laminectomy and (C) complete laminectomy of L4 spinous process in sequence. The intact group and partial laminectomy preserved the L3-L4 supraspinatous and Interspinous ligament; the complete laminectomy group removed the entire $L 4$ spinous process.

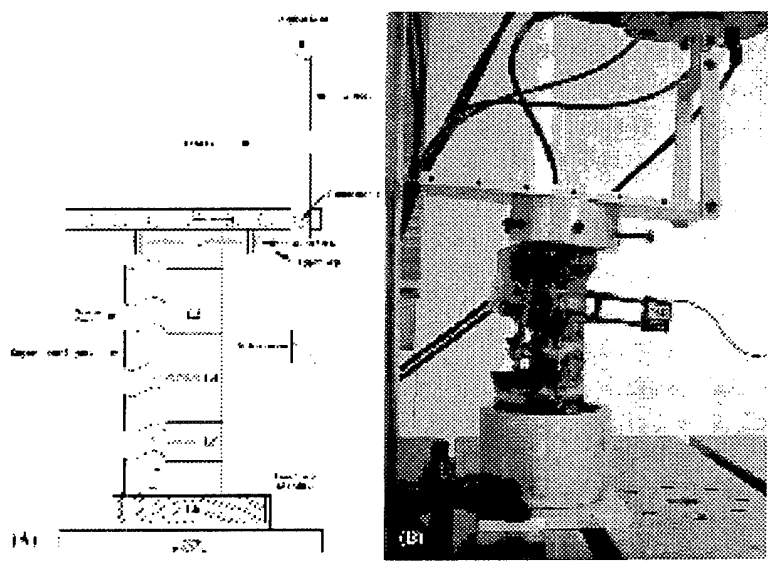

Fig. 2 The experimental setup (Left), and Flexion motion of instrumented lumbar spine (Right).

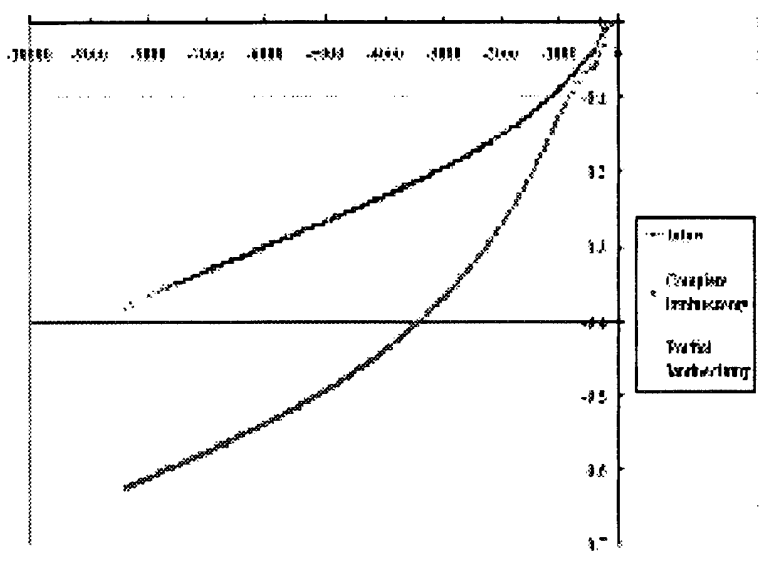

Fig. 3 The typical diagram of intervertebral displacement versus applied moment. The slop of Intact and Partial laminectomy is similar. The slop of Complete laminectomy is more steep.

Intact

Partial removal Complete removal Flexion Extension Flexion Extension Flexion Extension

\begin{tabular}{lllllll} 
Specimen 1 & 0.503 & 0.345 & 0.500 & 0.381 & 0.673 & 0.392 \\
Specimen 2 & 0.456 & 0.163 & 0.617 & 0.194 & 0.658 & 0.182 \\
Specimen 3 & 0.447 & 0.333 & 0.590 & 0.349 & 0.627 & 0.144 \\
Specimen 4 & 0.404 & 0.267 & 0.578 & 0.269 & 0.623 & 0.457 \\
Specimen 5 & 0.752 & 0.325 & 0.778 & 0.405 & 0.854 & 0.575 \\
Specimen 6 & 0.748 & 0.444 & 0.750 & 0.560 & 1.151 & 0.506 \\
Specimen 7 & 0.676 & 0.421 & 0.707 & 0.425 & 1.114 & 0.381 \\
Specimen 8 & 0.518 & 0.470 & 0.627 & 0.579 & 1.036 & 0.578 \\
\hline Ave. & 0.567 & 0.346 & 0.643 & 0.395 & 0.842 & 0.402 \\
S.D. & 0.138 & 0.101 & 0.094 & 0.131 & 0.228 & 0.164
\end{tabular}

Table 1. The intervertebral displacements on superior adjacent segment (L3-L4) for three different treatments of posterior complex. (Intact, Partial laminectomy, and Complete laminectomy). (Unit:mm)

\section{References}

[1] Kaneda K, Kazama H, Satoh S Fujiya M. Follow-up study of medial facetectomies and posterolateral fusion with instrumentation in unstable degenerative spondylolisthesis. Clin Orthop 1986; 203:159-67

[2] Chen WJ, Lai PL, Niu CC, Chen LH, Fu TS, Wong CB. Surgical treatment of adjacent instability after lumbar spine fusion. Spine 2001;E519-24

Corresponding author's e-mail: hardbone@yahoo.com 